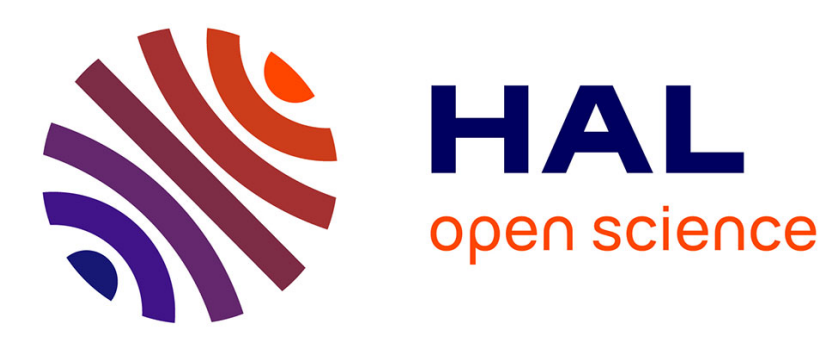

\title{
Design of ironless loudspeakers with ferrofluid seals: analytical study based on the coulombian model \\ Romain Ravaud, Guy Lemarquand
}

\section{To cite this version:}

Romain Ravaud, Guy Lemarquand. Design of ironless loudspeakers with ferrofluid seals: analytical study based on the coulombian model. Progress In Electromagnetics Research B, 2009, 14, pp.285-309. 10.2528/PIERB09031904 . hal-00377451

\section{HAL Id: hal-00377451 \\ https://hal.science/hal-00377451}

Submitted on 21 Apr 2009

HAL is a multi-disciplinary open access archive for the deposit and dissemination of scientific research documents, whether they are published or not. The documents may come from teaching and research institutions in France or abroad, or from public or private research centers.
L'archive ouverte pluridisciplinaire HAL, est destinée au dépôt et à la diffusion de documents scientifiques de niveau recherche, publiés ou non, émanant des établissements d'enseignement et de recherche français ou étrangers, des laboratoires publics ou privés. 


\title{
DESIGN OF IRONLESS LOUDSPEAKERS WITH FERROFLUID SEALS: ANALYTICAL STUDY BASED ON THE COULOMBIAN MODEL
}

\author{
R. Ravaud, G. Lemarquand
}

Laboratoire d'Acoustique de l'Universite du Maine, UMR CNRS 6613 Avenue Olivier Messiaen, 72085 Le Mans, France

E-mail : guy.lemarquand@univ-lemans.fr

\begin{abstract}
This paper presents an analytical method based on the coulombian model of a magnet for studying a ferrofluid seal in ironless electrodynamic loudspeakers. Such an approach differs from the ones generally used for studying such geometries because the ferrofluid used is submitted to a very high magnetic field. Consequently, its shape and its mechanical properties depend mainly on the magnetic field produced by the permanent magnets that constitute the ironless structure. The motor is constituted of outer stacked ring permanent magnets and the inner moving part is a piston. In addition, one ferrofluid seal is used for centering the moving part and ensuring the airtightness between the loudspeaker faces. The ferrofluid seal also exerts a pull back force on the moving piston. It is noted that this force depends on the lateral shape of the moving piston. Therefore, the piston profile is analytically studied in this paper. A peculiar attention is given to profiles that ensure the axial pull back force to be proportional to the piston displacement. Furthermore, a geometrical method is presented to design the shape of the ferrofluid seal according to the chosen piston profile. It can be noted that such a profile is elliptical in this study. Then, the magnetic energy of the ferrofluid seal is determined with the analytical expression of the magnetic energy density. Such an expression allows us to calculate the axial force created by the ferrofluid seal for a given profile.
\end{abstract}

\section{INTRODUCTION}

Three-dimensional analytical calculations of the magnetic field produced by ring or tile permanent magnets are generally used for the design of structures like axial or radial bearings and couplings. However, 
such analytical approaches can also be used for the study of ferrofluid seals submitted to very high magnetic fields. Strictly speaking, most studies dealing with ferrofluids are carried out by using several terms in the equation of equilibrium describing the ferrofluid [1]. However, not all these terms have the same weight and in some conditions, some of them can be omitted. This is particularly the case when a ferrofluid is submitted to a very high magnetic field. This often occurs when it is used in audio speakers [2] or ironless loudspeakers[3]-[5].

The first studies dealing with their behaviour under magnetic fields began in the early to mid-1960s [6]. Then, as ferrofluids have been improved, more and more engineering applications have been developed [7]. On the other hand, new three-dimensional approaches for calculating the magnetic field produced by permanent magnets have been proposed [8]-[20].

By using both the studies dealing with ferrofluids and the ones carried out by scientists involved in the three-dimensional calculations of the magnetic field produced by cylindrical geometries, some configurations ( as ironless loudspeakers ) can be investigated by using the concept of magnetic energy or magnetic pressure. Such terms are often simplified in many studies because they do not play a key role. However, in ironless loudspeakers, the precise determination of some terms (as the magnetic pressure) is required. Consequently, the study of the ferrofluid seal in ironless loudspeakers must be carried out with the three-dimensional analytical expressions of the magnetic field produced by ring permanent magnets.

The utility of ferrofluids lies in the fact that they can fulfil different functions: heat transfer, damping, motion control [21]-[25]. Ferrofluids can be used for the design of electromagnetic pumps for medical applications [26] or for the processing industry of liquid metal. Such fluids are also used for the design of bearings[27]-[30]. Other authors have studied high-speed magnetic fluid bearings and the properties of these ferrofluid seals when used in such applications[31]-[34]. An important parameter for the modeling ferrofluid seals is certainly its seal capacity [35] that can be used for optimization purposes. Furthermore, ferrofluids are also often used as squeeze films in bearings [36]-[38].

This paper describes a way of designing ferrofluid seals for ironless electrodynamic loudspeakers [39] with three-dimensional analytical expressions of the magnetic field created by ring permanent magnets. We can say that ferrofluid seals in such structures ensure the airtightness, 
play a heat transfer part and work as bearings. In this paper, the associated structure consists of both two outer ring permanent magnets and an inner moving piston. Thereby, the front face of this piston is the emissive surface. This paper presents ferrofluid seals associated with peculiar piston shapes which allow the axial displacement of the moving piston to be linear. Indeed, one of the ferrofluid seal properties is the axial force it creates on the moving piston. This force is linked to the profile of the piston lateral face. This paper describes the design of the piston shape which makes the axial force proportional to the piston displacement. Most of the calculations are analytical and the method used is based on a geometrical approach. The assumptions taken throughout this paper are based on the papers published by cunha [40] and matthies [41]. However, in this paper, the intensity of magnetization of a magnetic particle $(M)$ is saturated because the magnetic field we use is very high. Consequently, the relation between $M$ and $H$ is not linear. We neglect the aggregation in chains that occurs under very high magnetic fields [42] because this effect is not preponderant in the determination of the ferrofluid seal energy. In addition, $M$ is denoted $M_{s}$ in this paper. First, the design of the moving piston shape is studied. Then, the calculation of the axial force is discussed.

We can say that such an approach can be applied to the design of bearings using ferrofluid seals [43]-[45]. Indeed, the accurate knowledge of the quantity of ferrofluid, its shape or even the axial properties it can generate are an important element of information for the design of new types of ironless structures with permanent magnets and ferrofluid seals. In other words, this paper can be seen as an illustration of the use of three-dimensional analytical expressions for the design of ferrofluid seals. We think that it paves the way for a new approach for designing such structures.

\section{INTEREST OF CARRYING OUT THIS STUDY}

This study has been performed for the design of new structures of ironless loudspeakers. In our laboratory, we must find technological solutions for allowing the emissive piston to be proportional to its axial displacement. Some prototypes have been built and have shown promising sound reproductions. The back enclosure of an ironless loudspeaker functions as a pneumatic stiffness. Consequently, it generates a back pull force that is linear to its axial displacement. However, there are some temperature and pressure variations in the 
back enclosure. Small orifices can be performed in this enclosure to ensure pressure equilibrium between the two faces of the ironless loudspeaker but another pull back force is required for ensuring a good functioning of the emissive piston. We think that the piston shape can be optimized in a first step. We propose in this paper a fully theoretical study based on the coulombian model of a magnet that could be used for designing the piston profile.

This paper has two objectives. The first aim of this paper is to propose an original method, based on only geometrical considerations and magnetic energy calculations for studying the piston profile of an ironless loudspeaker. Some assumptions are taken into account but are fully justified by the very high magnetic fields produced by the ring permanent magnets. In addition, some experimental investigations have shown the accuracy of this model. The second objective of this paper is to show how exact three-dimensional analytical methods can be used for the design of ironless structures using ferrofluid seals. This is an important point because many devices use permanent magnets and ferrofluid seals. Nevertheless, the methods generally used for studying them are numerical. Even though these numerical methods are useful, the optimization of such structures with such approaches is rather difficult. The way we treat the problem in this paper is possible because the ferromagnetic particles are saturated and the Bernoulli equation can be reduced to a very simple form. However, it is emphasized that there are many devices that use saturated ferrofluid seals (as magnetic bearings).

\section{DESIGN OF THE PISTON}

\subsection{Geometry of the structure studied}

The structure studied consists of two outer ring permanent magnets, an inner non-magnetic piston, a ferrofluid seal placed in the air gap between the piston and the magnets and a back enclosure (Fig.1). Moreover, the emitting surface of the moving piston is plane. The two ring permanent magnets are stacked and their magnetizations are both radial, but in opposed directions. Furthermore, the ring permanent magnets have both the same dimensions and the same magnetization magnitude. The ring inner radius is $r_{i n}$, the ring outer radius is $r_{\text {out }}$ and the height of one ring permanent magnet is $h$. The non-magnetic piston is radially centered with the two ring permanent magnets. Moreover, the $z$ axis is an axis of symmetry. We can point out that the magnetic energy of the ferrofluid seal is determined with the analytical expression of the magnetic field created by the two ring permanent magnets. 


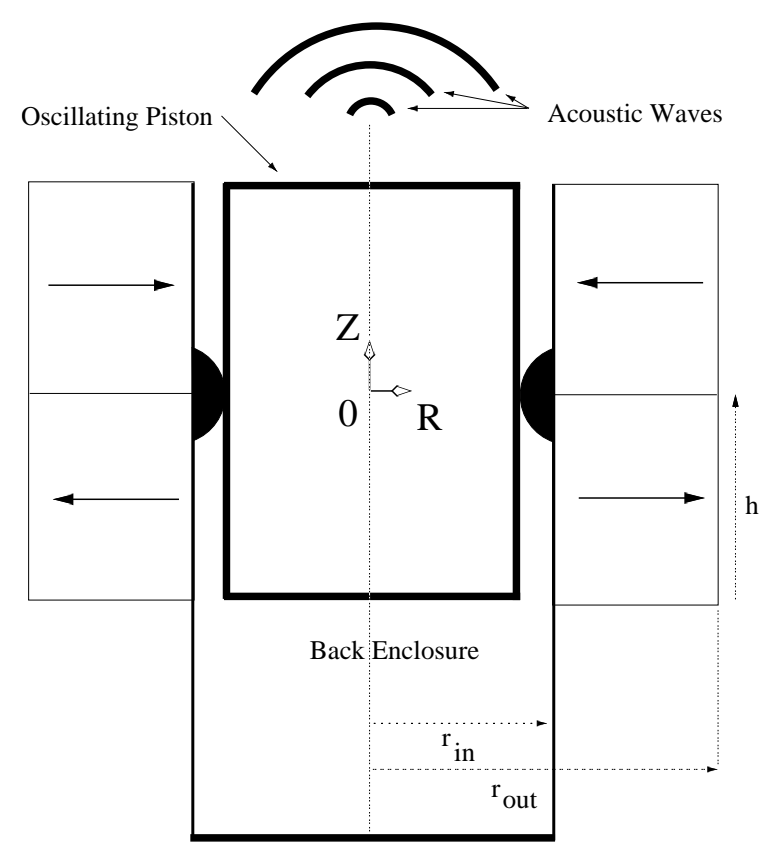

Figure 1. Geometry : both outer ring permanent magnets, the inner non-magnetic piston, the ferrofluid seal is placed in the air gap between the piston and the magnets; the ring inner radius is $r_{i n}$, the ring outer one is $r_{\text {out }}$ and the height of one ring permanent magnet is $h$. The inner piston is radially centered with the ring permanent magnets.

This is done by using the Coulombian model of permanent magnets. Consequently, each permanent magnet is represented by two curved planes. For the upper permanent magnet $(Z>0)$, the ring inner face $\left(R=r_{i n}\right)$ is charged with the magnetic pole surface density $+\sigma^{*}$ and the ring outer one $\left(R=r_{\text {out }}\right)$ is charged with the magnetic pole surface density $-\sigma^{*}$. For the lower permanent magnet $(Z<0)$, the ring inner face $\left(R=r_{i n}\right)$ is charged with the magnetic pole surface density $-\sigma^{*}$ and the ring outer one $\left(R=r_{\text {out }}\right)$ is charged with the magnetic pole surface density $+\sigma^{*}$. It is to be noted that, in Fig.1, the inner piston does not crush the ferrofluid seal. This structure is the starting point of this study. Then, the following step is to determine the optimal shape of the inner moving piston in order to have an axial pull back force proportional to the piston displacement. This optimal shape is obtained by using criteria based on the magnetic energy. The magnetic energy calculation of the ferrofluid seal is discussed in the next section. 


\subsection{Energy calculation of the ferrofluid seal}

The magnetic energy of the ferrofluid seal is based on several assumptions. First, the ferromagnetic particles are assumed small spheres which can be freely oriented in all the directions of space. Second, the particles are in the magnetic field created by the permanent magnets whose level is around 0.3 or $0.4 \mathrm{~T}$. Then, as the magnetic relative permeability of the ferrofluid is very small, the presence of ferrofluid does not modify the field created by the ring permanent magnets. In this paper, the maximal ferrofluid saturation magnetization $\mu_{0} M_{s}$ is $40 \mathrm{mT}$. Moreover, the ferrofluid volume is small and the maximal particle volume density is $5.5 \%$ : the magnetic field created by the particles themselves is small compared to the external field created by the permanent magnets. Consequently, this field is omitted. It can be noted that the magnetic interaction energy between the magnetic particles is important for dynamics studies. Indeed, it allows the magnetic particles both to be aligned between them and to form chains. However, we do not consider it because this study is a static one. Furthermore, the viscous effects and the magnetic interaction energy between the magnetic spherical particles are omitted. At least, the surface tension energy is not taken into account. We can point out that this latter assumption is also a strong one which can be justified by the fact that the magnetic field created by the ring permanent magnets is very high; the influence of the air on the surface tension can be neglected since the magnetic particles are extremely aligned with the strong magnetic field. Furthermore, the particles are assumed surfacted well enough so that they do not sediment in the high magnetic field created by the permanent magnets. In short, the only energy considered is the magnetic energy $E_{m}$ defined by (1):

$$
E_{m}=-\iiint_{(\Omega)} \mathbf{H}(r, z) \cdot \mu_{\mathbf{0}} \mathbf{M}_{\mathbf{s}} d V
$$

where $\mathbf{H}(r, z)$ is the magnetic field created by the two ring permanent magnets, $(\Omega)$ is the ferrofluid seal volume and $\mathbf{M}_{\mathbf{S}}$ is the intensity of magnetization. This expression is given in terms of elliptic integrals. The magnetic energy density $e_{m}(r, z)$ is given by $(2)$.

$$
e_{m}(r, z)=\mu_{0} M_{s} \sqrt{H_{r}(r, z)^{2}+H_{z}(r, z)^{2}}
$$

where $M_{s}$ is the intensity of magnetization of a magnetic particle. The radial component $H_{r}(r, z)$ and the axial component $H_{z}(r, z)$ have been published in [13] and these expressions have been reduced to compact forms in [8]. Consequently, we use the expressions determined by 
Babic and Akyel for studying the magnetic energy density $e_{m}(r, z)$. Let us consider a ring permanent magnet whose polarization is radial and directed towards the centre of the ring (0), the radial and axial components $h_{r}^{(1)}(r, z)$ and $h_{z}^{(1)}(r, z)$ are expressed as follows:

$$
\begin{aligned}
h_{r}^{(1)}(r, z)= & -\frac{J}{4 \pi \mu_{0}} \sum_{n=1}^{2}(-1)^{n-1} \frac{t_{n} k_{n}^{+}}{r} \sqrt{\frac{r_{\text {in }}}{r}}\left[K\left(k_{n}^{+}\right)+\frac{r-r_{\text {in }}}{r+r_{\text {in }}} \Pi\left(h^{+}, k_{n}^{+}\right)\right] \\
& +\frac{J}{4 \pi \mu_{0}} \sum_{n=1}^{2}(-1)^{n-1} \frac{t_{n} k_{n}^{-}}{r} \sqrt{\frac{r_{\text {out }}}{r}}\left[K\left(k_{n}^{-}\right)+\frac{r-r_{\text {out }}}{r+r_{\text {out }}} \Pi\left(h^{-}, k_{n}^{-}\right)\right] \\
h_{z}^{(1)}(r, z)= & \frac{J}{2 \pi \mu_{0}} \sum_{n=1}^{2}(-1)^{n-1} k_{n}^{+} \sqrt{\frac{r_{\text {in }}}{r}} K\left(k_{n}^{+}\right) \\
& -\frac{J}{2 \pi \mu_{0}} \sum_{n=1}^{2}(-1)^{n-1} k_{n}^{-} \sqrt{\frac{r_{\text {out }}}{r}} K\left(k_{n}^{-}\right)
\end{aligned}
$$

where

$$
\begin{array}{r}
k_{n}^{+}=\frac{4 r r_{\text {in }}}{\left(r+r_{\text {in }}\right)^{2}+t_{n}^{2}} \\
k_{n}^{-}=\frac{4 r r_{\text {out }}}{\left(r+r_{\text {out }}\right)^{2}+t_{n}^{2}} \\
h^{+}=\frac{4 r r_{\text {in }}}{\left(r+r_{\text {in }}\right)^{2}} \\
h^{-}=\frac{4 r r_{\text {out }}}{\left(r+r_{\text {out }}\right)^{2}} \\
t_{1}=z-z_{2} \\
t_{2}=z-z_{1}
\end{array}
$$

where $J$ is the polarization of the ring permanent magnet, $h=z_{2}-z_{1}$ is its height, $\mu_{0}$ is the permeability of the vacuum, $K(k), E(k)$ and $\Pi(h, k)$ are the complete elliptic integrals of the first, second and third kind. In our configuration, we use two ring permanent magnets with opposite magnetizations. The total magnetic field produced by these two ring permanent magnets can be easily obtained by using the principle of superposition. By denoting $h_{r}^{(2)}(r, z)$ and $h_{z}^{(2)}(r, z)$ the radial and axial components of the magnetic field produced by the other ring permanent magnet, the total magnetic field is:

$$
\vec{H}(r, z)=\left(h_{r}^{1}(r, z)+h_{r}^{2}(r, z)\right) \vec{u}_{r}+\left(h_{z}^{1}(r, z)+h_{z}^{2}(r, z)\right) \vec{u}_{z}
$$




$$
=H_{r}(r, z) \vec{u}_{r}+H_{z}(r, z) \vec{u}_{z}
$$

in which $H_{r}(r, z)$ and $H_{z}(r, z)$ are used in (1) for calculating the magnetic energy density.

The magnetic field created by the two ring permanent magnets depends only on both radius $r$ and altitude $z$ since the azimuthal component equals zero because of the cylindrical symmetry. The components of this field have been established in previous papers [13][15]. It can be noted that in most of the papers dealing with the modeling of ferrofluids, many parameters are taken into account in the Bernoulli equation but the magnetic field is always very simplified. Indeed, the magnetic field is often assumed constant. In this paper, the way of studying the ferrofluid seal is different. Indeed, we propose to study a complex geometry with an analytical expression of the magnetic field. Consequently, the term which is the most important in the Bernoulli equation is exact in this paper. This way of optimizing a piston profile is very useful in such geometry because it has a very low computational cost. Moreover, it allows making easily parametric studies on the configuration dimensions.

\subsection{Determination of the piston optimal shape}

The moving part of electrodynamic loudspeakers must be axially stable in order to avoid being ejected. This remains true for ironless electrodynamic loudspeakers and a good axial stability of the moving piston is thus searched. We can point out that such a stability is reached thanks to the pull back force exerted by the ferrofluid seal on the piston. It can be noted that the greater this force is, the better the axial stability is. However, if the force intensity is too high, the piston movement can be impeded. As a consequence, some compromises must be made. In any case, it is useful to control the force variation when the piston moves. Therefore, the design of the inner piston is made so that the variation force is linear. For this purpose, the lateral face of the piston must be considered. As the variation force depends on its profile, the piston shape is determined by using a magnetic energy criterion of the ferrofluid seal. To do so, the magnetic energy in the ferrofluid seal is first calculated when the inner piston does not crush the ferrofluid. The value obtained is denoted $E_{m}[1]$. Second, the same geometry is considered but the piston has a larger diameter and it crushes the ferrofluid seal (Fig. 2). The magnetic energy in the ferrofluid is determined in this configuration and the obtained value is denoted $E_{m}[2]$. Furthermore, the pull back force is defined by (6).

$$
F=\frac{E_{m}[1]-E_{m}[2]}{\Delta r}
$$




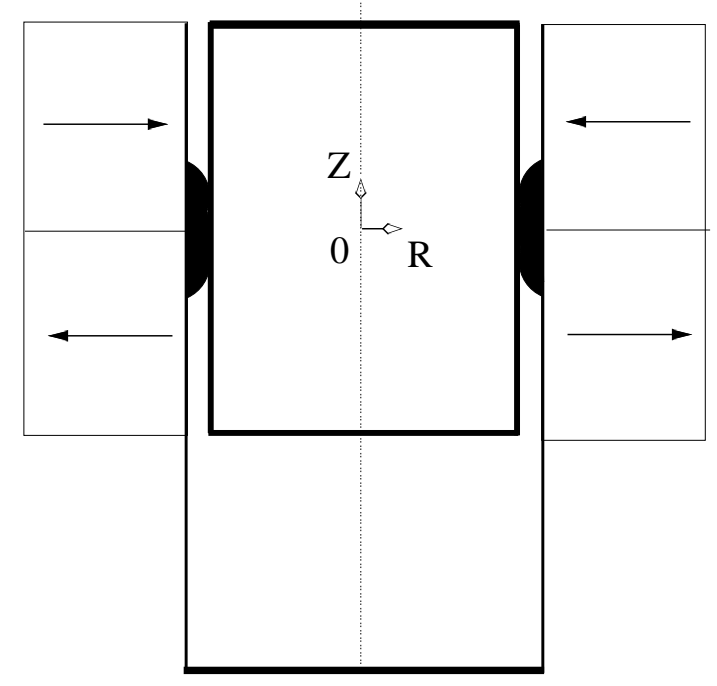

Figure 2. Geometry : two outer ring permanent magnets, an inner non-magnetic piston whose radius is larger than the previous one and a ferrofluid seal placed between them; the ring inner radius is $r_{i n}$ and the height of one ring permanent magnet is $h$. The inner piston is radially centered.

where $\Delta r$ represents the increase in the piston radius. These values are qualitatively represented in Fig.3. The magnetic energy of the ferrofluid seal is represented as a function of the piston radius variation. Figure 3 shows that $E_{m}[2]$ is smaller than $E_{m}[1]$. Indeed, the increase in the piston diameter generates the crushing of the ferrofluid seal and consequently a diminution in the magnetic energy. Furthermore, Fig.3 clearly shows that the relationship between the axial pull back force and the axial displacement of the moving piston depends on the piston radius, and so, on the way the piston crushes the ferrofluid seal. In order to have a good axial stability, the pull back force should increase when the axial displacement of the piston increases. Therefore, the piston radius must not be constant, but should vary along the $Z$ axis. In addition, its radius should increase with an increasing altitude $z$. Figure 4 shows such a geometry with a piston radius which depends on $z$. It can be noted that the relation linking the piston radius and the altitude depends on both the ferrofluid quantity and the structure dimensions. Thereby, the nature of this relation has an impact on the intensity of the axial pull back force. If we look for a great pull 


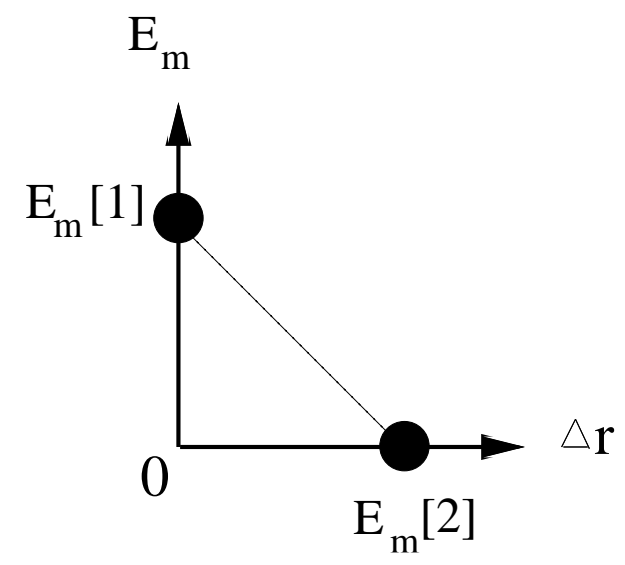

Figure 3. Qualitative representation of the magnetic energy of the ferrofluid seal in two configurations; $E_{m}[1]$ is the energy when the piston does not crush the ferrofluid seal. $E_{m}[2]$ is the energy when the piston crushes the ferrofluid seal, that is, when its radius increased by $\Delta r$.

back force, the piston radius variation must be important with the altitude $z$ (Fig.5). As the pull back force also impedes the piston movement, an optimal piston shape piston must be determined for a given ferrofluid quantity. In fact, several shapes have been tried out for a given ferrofluid quantity. In conclusion, the piston shape that gives a linear pull back force is an ellipse. It is noted that the equation of the ellipse depends on the ferrofluid quantity used.

\section{CALCULATION OF THE PULL BACK FORCE}

The optimal shape of the piston is an ellipse and its axial stability is now studied. Consequently, the pull back force must be calculated. For this purpose, a method based on the determination of the magnetic energy of the ferrofluid is presented. Two configurations are considered. The ferrofluid seal energy is first determined when the piston is axially centered and newt when the piston has moved axially. It is noted that the second configuration corresponds to the case when the piston is no longer axially centered. As the piston remains radially centered over this study, the whole structure remains axisymmetrical. Therefore, the volume of ferrofluid is totally characterized by the surface of its cross-section in an axial plane. The method consists in geometrical reflections on this surface and its variations. Thus, the 


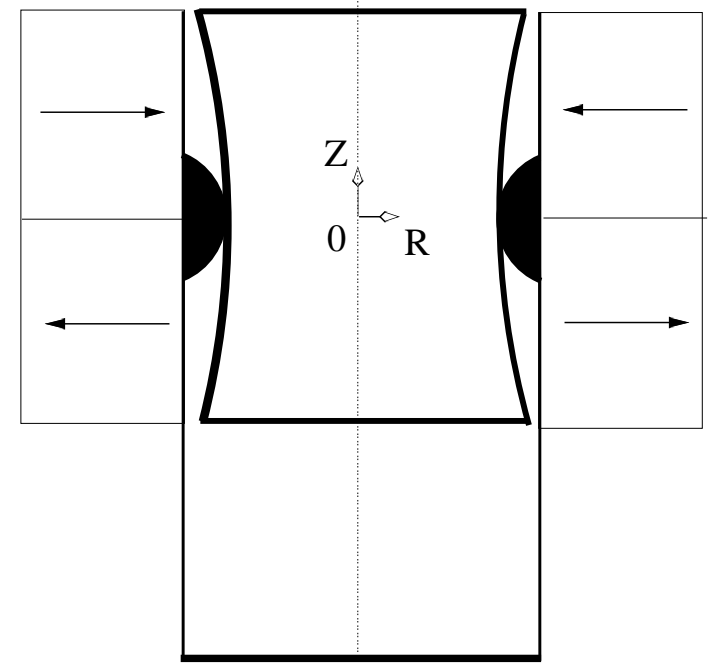

Figure 4. Geometry : two outer ring permanent magnets and an inner non-magnetic piston whose radius depends 'smoothly' on the altitude $z$.

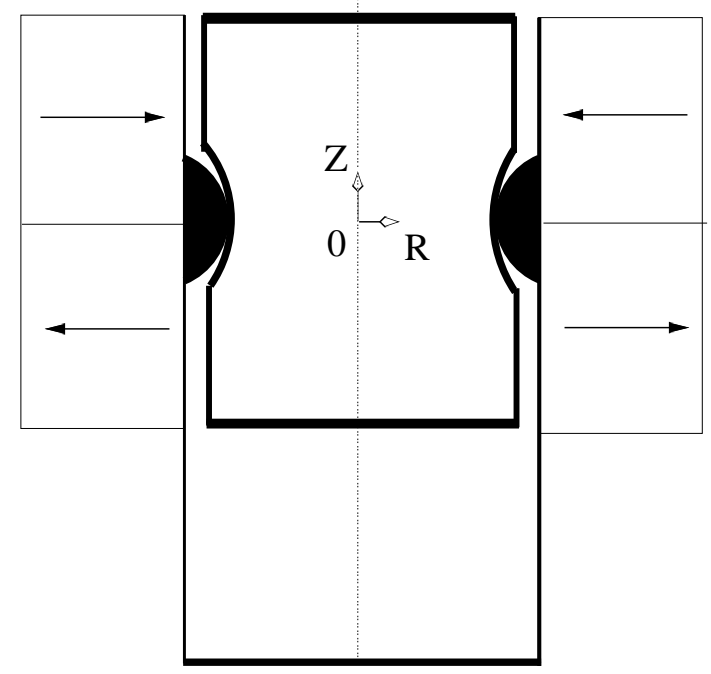

Figure 5. Geometry : two outer ring permanent magnets and an inner non-magnetic piston whose radius depends 'sharply' on the altitude $z$. 
ferrofluid quantity is described in terms of surface or of contour in this plane.

\subsection{Determination of the ferrofluid seal energy when the piston is axially centered}

\subsubsection{Ferrofluid seal free shape}

A small quantity of ferrofluid is used to make the seal. In absence of the piston, the seal has its free shape and its contour can be described approximately in terms of an equation of ellipse (7). This equation has been determined as follows: we have drawn the magnetic lines created by the ring permanent magnets and have looked for a simple equation which can describes the magnetic lines for small quantities of ferrofluids. The error done is inferior to $2 \%$. As a consequence, we can approximate the contour with (7).

$$
\frac{\left(r-r_{f 1}\right)^{2}}{a_{1}^{2}}+\frac{\left(z-z_{f 1}\right)^{2}}{b_{1}^{2}}=1
$$

where $a_{1}, b_{1}, r_{f 1}$ and $z_{f 1}$ are given in Table 1 . Such an ellipse is represented in Fig. 6 where the scale of the $R$ axis is magnified. The analytical integration of (8) gives the value of the ferrofluid seal crosssection:

$$
S_{\text {free }}=2 \int_{r=r_{0}}^{r=r_{i n}}\left(b_{1} \sqrt{1-\frac{\left(r-r_{f 1}\right)^{2}}{a_{1}^{2}}}\right) d r
$$

\subsubsection{Superposition of the piston contour and of the ferrofluid seal free contour}

The presence of the non-magnetic piston changes the seal geometrical shape, as the fluid fits the piston contour. The piston profile chosen is a portion of an ellipse (Fig. 7). This profile has not been obtained directly but is the result of several attempts. Indeed, the final aim of this study being to have a pull back force proportional to the axial displacement of the piston, its geometry is the most important parameter.

The piston minimal radius is $r_{\min }$, its maximum one is $r_{\max }$. The dependence of the piston radius with the altitude $z$ is given by the following equation of ellipse (9):

$$
\frac{\left(r-r_{f 2}\right)^{2}}{a_{2}^{2}}+\frac{(z)^{2}}{b_{2}^{2}}=1
$$






Figure 6. Representation of the ferrofluid seal free shape in an axial plane: the scale is magnified along the $R$ axis. The ferrofluid volume is proportional to the streaked surface $\left(S_{\text {free }}\right)$.

\begin{tabular}{lc}
\hline \hline Parameter & Value \\
\hline$a_{1}$ & 0,00025 \\
$b_{1}$ & 0,000275 \\
$r_{f 1}$ & 0,025 \\
$a_{2}$ & 0,00025 \\
$b_{2}$ & 0,001 \\
$r_{f 2}$ & 0,0251 \\
$a_{3}$ & 0,0003091 \\
$b_{3}$ & 0,00034 \\
$r_{3}$ & 0,0249 \\
$a_{4}$ & 0,00025 \\
$b_{4}$ & 0,001 \\
$r_{f 4}$ & 0,0251 \\
$z_{f 4}$ & 0,000252 \\
$a_{5}$ & 0,000309 \\
$b_{5}$ & 0,00034 \\
$r_{f 5}$ & 0,0249005 \\
$r_{7}$ & 0,0248509 \\
$r_{10}$ & 0,0248985 \\
\hline \hline
\end{tabular}

Table 1. Parameters 


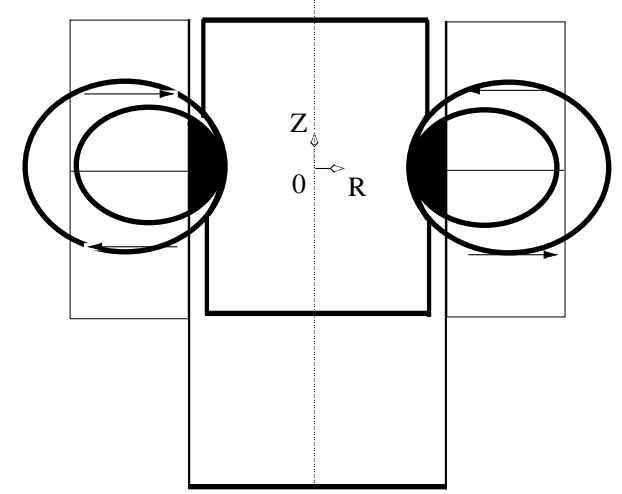

Figure 7. Illustration of the shapes of the piston and of the seal: both profiles are portion of ellipses. The scale along the $R$ axis is not magnified here

where $r_{f 2}, a_{2}$ and $b_{2}$ are given in Table 1 .

Figure 8 shows the superposition of both the piston profile and the shape of the free ferrofluid seal. The points of intersection of both contours are denoted $P_{1}$ and $P_{2}$. The left hand streaked surface, $S_{\text {excess }}$, represents the quantity of ferrofluid which will move because of the presence of the piston.

\subsubsection{Determination of the shape of the ferrofluid seal in presence of} the axially centered piston

In presence of the piston, the ferrofluid seal no longer has its free shape, but instead, it fits the piston own shape. Figure 8 shows that a quantity of ferrofluid has therefore to move. This quantity is proportional to the left hand streaked surface $S_{\text {excess }}$ and can be analytically determined by $(10)$.

$$
\begin{aligned}
S_{\text {excess }}= & 2\left(\int_{r=r_{0}}^{r=r_{2}}\left(b_{1} \sqrt{1-\frac{\left(r-r_{f 1}\right)^{2}}{a_{1}^{2}}}\right)\right) d r \\
& -2\left(\int_{r=r_{\min }}^{r=r_{2}}\left(b_{2} \sqrt{1-\frac{\left(r-r_{f 2}\right)^{2}}{a_{2}^{2}}}\right) d r\right)
\end{aligned}
$$

This "exceeding" ferrofluid quantity moves towards the remaining free space between the piston and the magnets and thickens symmetrically 


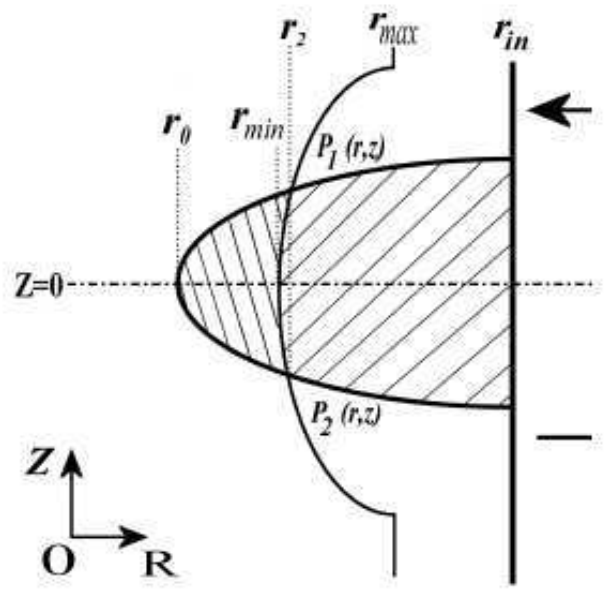

Figure 8. Ferrofluid seal in presence of the axially centered piston: the left hand streaked surface represents the quantity of ferrofluid which will move.

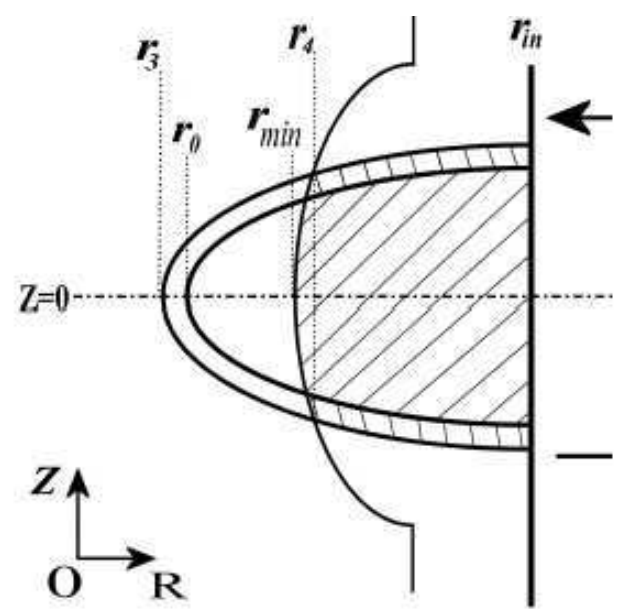

Figure 9. Determination of the ferrofluid seal contour in presence of the axially centered piston

the existing seal. The new contour is also described in terms of an equation of ellipse (Fig. 9) whose coefficients $a_{3}, b_{3}$ and $r_{3}$ must be determined so that (11) is verified. 


$$
\begin{aligned}
S_{\text {ferro }}= & 2\left(\int_{r=r_{\min }}^{r=r_{3}}\left(b 2 \sqrt{1-\frac{\left(r-r_{f 2}\right)^{2}}{a_{2}^{2}}}\right)\right) d r \\
& +2\left(\int_{r=r_{3}}^{r=r_{i n}}\left(b 3 \sqrt{1-\frac{\left(r-r_{f 1}\right)^{2}}{a_{3}^{2}}}\right) d r\right)
\end{aligned}
$$

where $S_{\text {ferro }}$ is the surface of the new ferrofluid seal cross-section. The parameter $r_{f 1}$ is kept in the second term of the second member of (11) because of the equation of ellipse describing the piston contour. Moreover, the coefficients $a_{3}, b_{3}$ and $r_{3}$ can be determined by a dichotomy search by taking into account the fact that the transformation between (7) and (13) is isometric. Consequently, the following relation must be verified (12):

$$
\frac{a_{1}}{b_{1}}=\frac{a_{3}}{b_{3}}
$$

As a result, the equation of ellipse describing the ferrofluid seal contour between $r_{3}$ and $r_{i n}$ in presence of the inner non-magnetic piston is given by (13):

$$
\frac{\left(r-r_{f 1}\right)^{2}}{a_{3}^{2}}+\frac{(z)^{2}}{b_{3}^{2}}=1
$$

where $a_{3}, b_{3}$ and $r_{3}$ are given in Table 1 . The ferrofluid seal shape is represented in Fig. 10.

\subsubsection{Energy of the ferrofluid seal}

As we exactly know where the ferrofluid moves when the inner non-magnetic piston is present, the energy in the ferrofluid can be calculated. Furthermore, the energy $E_{1}$ of the ferrofluid seal with an axially centered piston can be determined in two steps. The first step consists in calculating the magnetic energy in the ferrofluid located between $r_{3}$ and $r_{i n}$. This magnetic energy, $E m_{1}$, is given by (14).

$$
\begin{aligned}
E m_{1}= & \int_{\theta=0}^{\theta=2 \pi} \int_{z=z_{\text {inf }}}^{z=z_{\text {sup }}} \int_{r=r_{4}}^{r=r_{\text {in }}} e_{m}(r, z) r d r d \theta d z \\
& -\int_{\theta=0}^{\theta=2 \pi} \int_{z=z_{\text {inf }}}^{z=z_{\text {sup }}} \int_{r=r_{4}}^{r=r_{3}} e_{m}(r, z) r d r d \theta d z
\end{aligned}
$$

The second step consists in determining the magnetic energy in the ferrofluid located between $r_{\min }$ and $r_{3}$. For this purpose, a nonlinear regression allows us to approximate the contour of the ferrofluid 


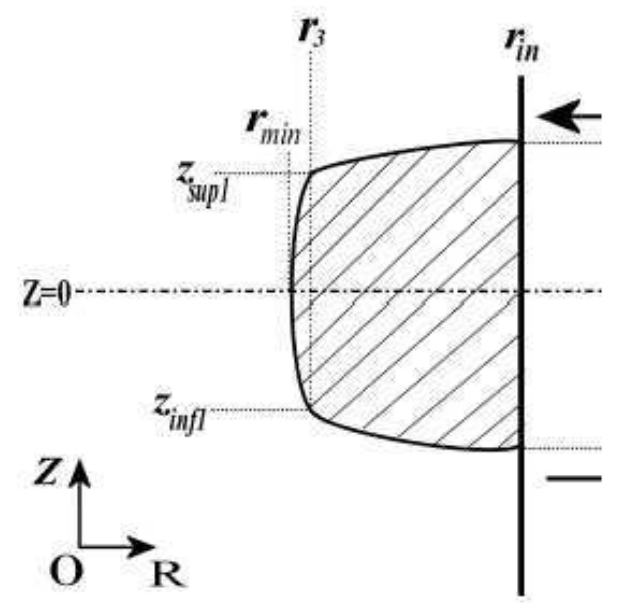

Figure 10. Shape of the ferrofluid seal in presence of the inner nonmagnetic piston

seal between $r_{\min }$ and $r_{3}$ by a parabolic equation. Consequently, the integration limits are not constant but depend on the altitude $z$. The lower limit $r(z)$ verifies (15).

$$
r(z)=p_{1} z^{2}+p_{2} z+p_{3}
$$

The parameters $p_{1}, p_{2}$ and $p_{3}$ appear in the integration limits of the energy volume density. Therefore, the magnetic energy $E_{m 2}$ of the ferrofluid located between $r_{\min }$ and $r_{3}$ is given by (16):

$$
E m_{2}=\int_{\theta=0}^{\theta=2 \pi} \int_{\left(p_{1} z^{2}+p_{2} z+p_{3}\right)}^{r_{3}} \int_{z_{\text {inf } 1}}^{z_{\text {sup } 1}} e_{m}(r, z) r d r d \theta d z
$$

The total magnetic energy $E_{1}$ in the ferrofluid seal is the sum of both partial energies (17):

$$
E_{1}=E m_{1}+E m_{2}
$$

\subsection{Determination of the ferrofluid seal energy when the inner piston is axially decentered}

4.2.1. Superposition of the piston contour and of the ferrofluid seal free contour

When the piston moves, it becomes axially decentered as represented in Fig. 11. The method remains the same as when the piston is centered 


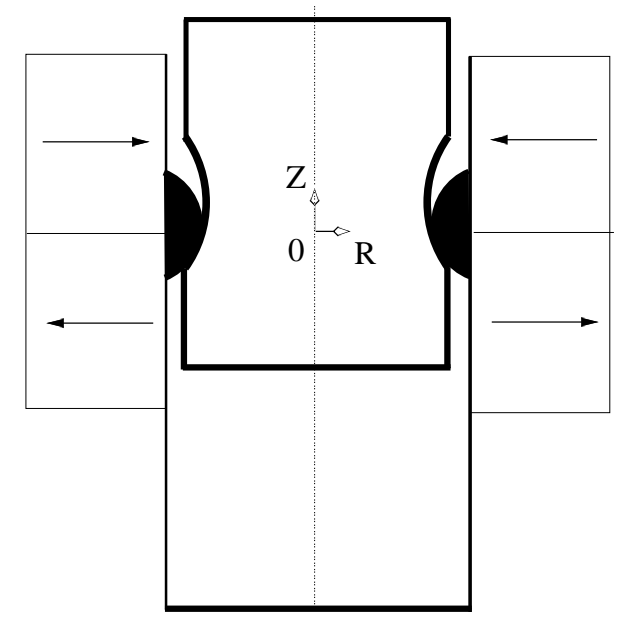

Figure 11. Geometry : two outer ring permanent magnets and an inner non-magnetic piston with a ferrofluid seal between them. The inner piston is not axially centered.

but calculations become more difficult. Indeed, even if ferrofluid seal free contour remains the same, the piston contour is no longer symmetrically superposed and the points of intersection determination of both contours, $P_{5}(r, z)$ and $P_{6}(r, z)$, is more complicated (Fig. 12). The equation of ellipse describing the axially decentered piston is given by (18):

$$
\frac{\left(r-r_{f 4}\right)^{2}}{a_{4}^{2}}+\frac{\left(z-z_{f 4}\right)^{2}}{b_{4}^{2}}=1
$$

where $r_{f 4}, a_{4}, b_{4}$ and $z_{f 4}$ are given in Table 1 . The points of intersection of (7) and (18) can thus be determined.

\subsubsection{Determination of the shape of the ferrofluid seal in presence of the axially decentered piston}

As previously, in presence of the piston, the ferrofluid fits the piston shape. The "exceeding" ferrofluid quantity appearing in the left hand streaked surface in Fig. 12 moves towards the remaining free space between the piston and the magnets. Consequently, it thickens the existing seal and the new contour is also described in terms of an equation of ellipse. The total quantity of ferrofluid is still the same. In addition, the seal shape is not symmetrical according to the $R$ axis and the points of intersection $P_{7}\left(r_{7}, z_{7}\right)$ and $P_{8}\left(r_{10}, z_{10}\right)$ (Fig. 13)must 


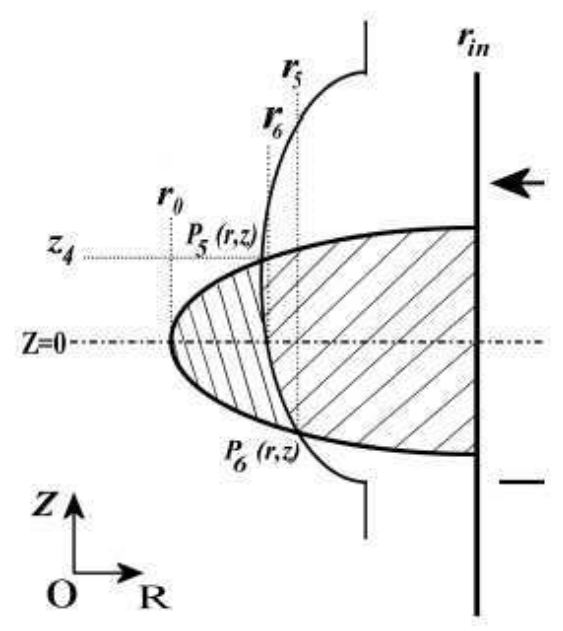

Figure 12. Ferrofluid seal in presence of the axially decentered piston: the left hand streaked surface represents the quantity of ferrofluid which will move.

be found so that (19) is verified.

$$
\begin{aligned}
S_{\text {ferro }}= & 2 \int_{r=r_{10}}^{r=r_{i n}}\left(b_{5} \sqrt{1-\frac{\left(r-r_{f 5}\right)^{2}}{a_{5}^{2}}}\right) d r \\
& +\int_{r=r_{9}}^{r_{10}}\left(b_{5} \sqrt{1-\frac{\left(r-r_{f 5}\right)^{2}}{a_{5}^{2}}}\right) d r \\
& -\int_{r=r_{9}}^{r=r_{1}}\left(b_{5} \sqrt{1-\frac{\left(r-r_{f 5}\right)^{2}}{a_{5}^{2}}}\right) d r \\
& -\left(r_{10}-r_{1}\right) z_{7} \\
& +\int_{r=r_{1}}^{r=r_{10}}\left(b_{4} \sqrt{1-\frac{\left(r-r_{f 4}\right)^{2}}{a_{4}^{2}}}\right) d r
\end{aligned}
$$

where $r_{f 5}, a_{5}, b_{5}, r_{7}$ and $r_{10}$ are given in Table 1 . These coefficients are obtained by a multivariable dichotomy search. It is to be noted that the equation of ellipse describing the ferrofluid seal contour in presence of the axially decentered piston is the same as the one describing the ferrofluid seal contour in presence of the axially centered piston. The final shape of the ferrofluid seal in presence of the axially decentered 


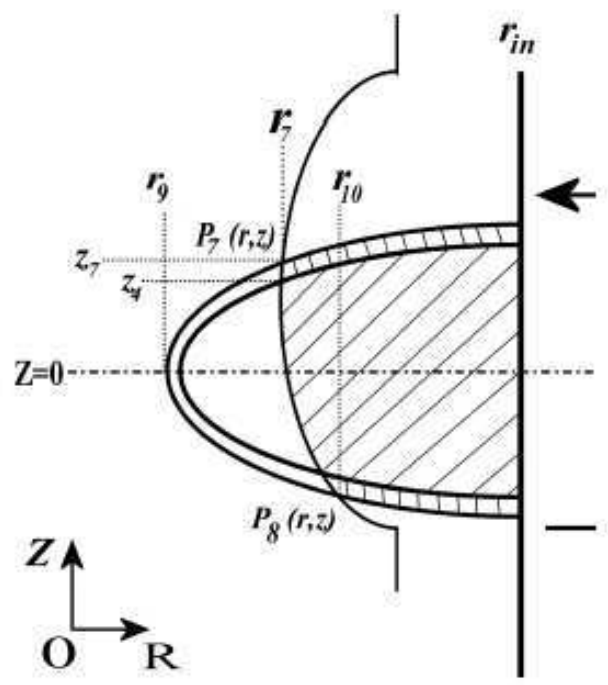

Figure 13. Determination of the ferrofluid seal contour in presence of the axially decentered piston

piston is shown in Fig. 14.

4.2.3. Magnetic energy of the ferrofluid seal in presence of the axially decentered piston.

The magnetic energy of the ferrofluid can be calculated in two steps. The first step consists in calculating the magnetic energy $E_{m 3}$ of the ferrofluid located between $r_{10}$ and $r_{i n}$ with (20).

$$
\begin{aligned}
E_{m 3}= & \int_{\theta=0}^{\theta=2 \pi} \int_{z=z_{\text {inf } 2}}^{z=z_{\text {sup } 2}} \int_{r=r_{1}}^{r=r_{i n}} e_{m}(r, z) r d r d \theta d z \\
& -\int_{\theta=0}^{\theta=2 \pi} \int_{z=z_{\text {inf } 2}}^{z=z_{\text {sup } 2}} \int_{r=r_{1}}^{r=r_{10}} e_{m}(r, z) r d r d \theta d z
\end{aligned}
$$

The second step consists in calculating the magnetic energy $E_{m 4}$ of the ferrofluid located between $r_{7}$ and $r_{10}$. For this purpose, a non linear regression allows us to approximate the contour of the ferrofluid seal between $r_{7}$ and $r_{10}$ by a parabolic equation. Consequently, the integration limits are not constant but depend on the altitude $z$. The 


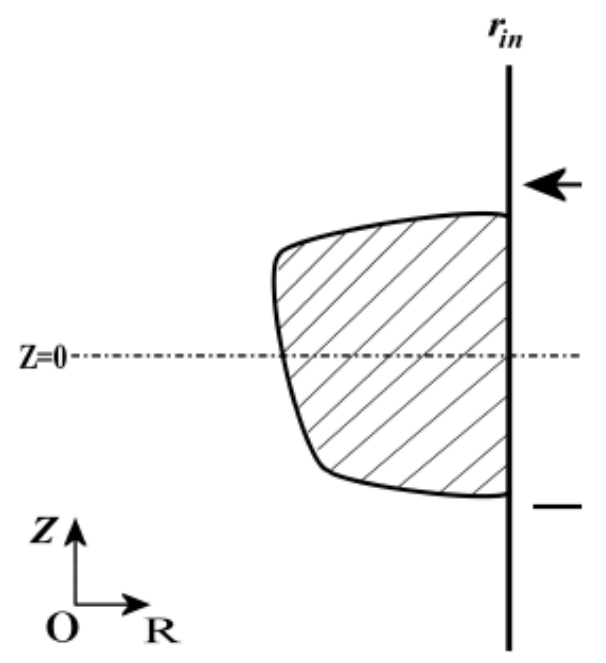

Figure 14. Shape of the ferrofluid seal when the inner piston is not axially centered

lower limit $r(z)$ verifies (21).

$$
r(z)=p_{4} z^{2}+p_{5} z+p_{6}
$$

It is noted that the parameters $p_{4}, p_{5}$ and $p_{6}$ appear in the integration limits of the energy volume density. Therefore, the magnetic energy $E_{m 4}$ of the ferrofluid seal between $r_{7}$ and $r_{10}$ is given by (22).

$$
E m_{4}=\int_{\theta=0}^{\theta=2 \pi} \int_{\left(p_{4} z^{2}+p_{5} z+p_{6}\right)}^{r_{10}} \int_{z_{\text {inf } 2}}^{z_{\text {sup } 2}} e_{m}(r, z) r d r d \theta d z
$$

where $z_{\text {inf } 2}$ and $z_{\text {sup } 2}$ correspond to the points of intersection between the line of equation $r=r_{10}$ and the parabolic equation $r=p_{4} z^{2}+$ $p_{5} z+p_{6}$. As a result, the magnetic energy of the ferrofluid seal is given by $(23)$.

$$
E_{2}=E_{m 3}+E_{m 4}
$$

\subsubsection{Determination of the axial force exerted by the ferrofluid seal}

The determination of the magnetic energy in the two previous configurations allows us to calculate the axial pull back force $F_{\text {axial }}$ of the ferrofluid seal with (24).

$$
F_{\text {axial }}=\frac{E_{2}-E_{1}}{\Delta z}
$$




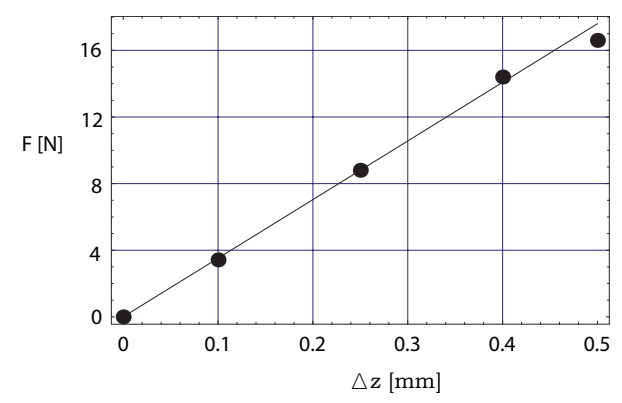

Figure 15. Pull-back force exerted by the seal when the piston has an elliptic profile versus the piston displacement.

where $E_{1}$ and $E_{2}$ have been determined previously. It is noted that all the energy density calculations are done analytically and the energy calculations are done numerically.

When the numerical set of values given in Table 1 is considered, the calculation of the force exerted by the seal on the piston for several axial displacements of the piston is shown in Fig.15. This proves that an elliptical piston profile effectively leads to linear force variations with the piston displacement. The corresponding axial stiffness is approximately $3.6 \mathrm{~N} / \mathrm{mm}$.

\section{CONCLUSION}

This paper has described the use of ferrofluid seals in ironless electrodynamic loudspeakers by using the three-dimensional analytical expressions of the magnetic field created by ring permanent magnets. One of the seal properties lies in the pull back force it exerts on the moving piston. It is noted that this force depends on both the ferrofluid quantity used and the piston lateral profile. Moreover, this paper shows that the piston shape can be chosen to control the force variation with the axial displacement. For small ferrofluid quantities, an elliptical piston profile leads to a pull back force which varies linearly with the piston axial displacement. Furthermore, this paper also describes a semi-analytical method for calculating the pull back force. Such an approach is based on geometrical reflections enabled by the axisymmetry of the device. Thereby, the shape of the ferrofluid seal can be found and the energy in the ferrofluid calculated for each piston position. Then, the force exerted by the seal on the piston is deducted. 
Such an approach can be very useful to determine the mechanical properties of ferrofluid seals in several engineering applications. In addition, we think that such an approach paves the way for new analytical approaches for the design of airtightness seals or bearings using ferrofluid seals.

\section{REFERENCES}

1. K. Raj, V. Moskowitz, and R. Casciari, "Advances in ferrofluid in ferrofluid technology," Journal of Magnetism and Magnetic Materials, vol. 149, pp. 174-180, 1995.

2. R. E. Rosensweig, Y. Hirota, S. Tsuda, and K. Raj, "Study of audio speakers containing ferrofluid," J. Phys. : Condens. Matter, vol. 20, 2008.

3. G. Lemarquand, "Ironless loudspeakers," IEEE Trans. Magn., vol. 43, no. 8, pp. 3371-3374, 2007.

4. R. Ravaud and G. Lemarquand, "Modelling an ironless loudspeaker by using three-dimensional analytical approaches," Progress in Electromagnetics Research, PIER 91, pp. 53-68, 2009.

5. R. Ravaud and G. Lemarquand, "Mechanical properties of a ferrofluid seal: three-dimensional analytical study based on the coulombian model," Progress in Electromagnetics research B, vol. 13, pp. 385-407, 2009.

6. R. E. Rosensweig, Ferrohydrodynamics. Dover, 1997.

7. Y. L. Raikher, V. I. Stepanov, J. C. Bacri, and R. Perzynski, "Orientational dynamics in magnetic fluids under strong coupling of external and internal relaxations," Journal of Magnetism and Magnetic Materials, vol. 289, pp. 222-225, 2005.

8. S. Babic and C. Akyel, "Improvement in the analytical calculation of the magnetic field produced by permanent magnet rings," Progress in Electromagnetics Research C, vol. 5, pp. 71-82, 2008.

9. S. Babic and C. Akyel, "An improvement in the calculation of the magnetic field for an arbitrary geometry coil with rectangular cross section," International Journal of Numerical Modelling: Electronic Networks, Devices and Fields, vol. 18, pp. 493-504, November 2005.

10. S. Babic and C. Akyel, "Magnetic force calculation between thin coaxial circular coils in air," IEEE Trans. Magn., vol. 44, no. 4, pp. 445-452, 2008.

11. S. Babic, C. Akyel, and M. M. Gavrilovic, "Calculation improvement of $3 \mathrm{~d}$ linear magnetostatic field based on fictitious 
magnetic surface charge," IEEE Trans. Magn., vol. 36, no. 5, pp. 3125-3127, 2000.

12. S. Babic, C. Akyel, and S. Salon, "New procedures for calculating the mutual inductance of the system: filamentary circular coilmassive circular solenoid," IEEE Trans. Magn., vol. 39, no. 3, pp. 1131-1134, 2003.

13. R. Ravaud, G. Lemarquand, V. Lemarquand, and C. Depollier, "Analytical calculation of the magnetic field created by permanent-magnet rings," IEEE Trans. Magn., vol. 44, no. 8, pp. 1982-1989, 2008.

14. R. Ravaud, G. Lemarquand, V. Lemarquand, and C. Depollier, "Discussion about the analytical calculation of the magnetic field created by permanent magnets.," Progress in Electromagnetics Research B, vol. 11, pp. 281-297, 2009.

15. R. Ravaud, G. Lemarquand, V. Lemarquand, and C. Depollier, "The three exact components of the magnetic field created by a radially magnetized tile permanent magnet.," Progress in Electromagnetics Research, PIER 88, pp. 307-319, 2008.

16. R. Ravaud and G. Lemarquand, "Analytical expression of the magnetic field created by tile permanent magnets tangentially magnetized and radial currents in massive disks," Progress in Electromagnetics Research B, vol. 13, pp. 309-328, 2009.

17. R. Ravaud and G. Lemarquand, "Discussion about the magnetic field produced by cylindrical halbach structures," Progress in Electromagnetics Research B, vol. 13, pp. 275-308, 2009.

18. R. Ravaud, G. Lemarquand, V. Lemarquand, and C. Depollier, "Magnetic field produced by a tile permanent magnet whose polarization is both uniform and tangential," Progress in Electromagnetics Research B, vol. 13, pp. 1-20, 2009.

19. J. P. Selvaggi, S. Salon, O. M. Kwon, and M. V. K. Chari, "Calculating the external magnetic field from permanent magnets in permanent-magnet motors - an alternative method," IEEE Trans. Magn., vol. 40, no. 5, pp. 3278-3285, 2004.

20. R. Ravaud, G. Lemarquand, V. Lemarquand, and C. Depollier, "Torque in permanent magnet couplings: comparison of uniform and radial magnetization," Journal of applied physics, vol. 105, no. 5, 2009.

21. J. Bajkowski, J. Nachman, M. Shillor, and M. Sofonea, "A model for a magnetorheological damper," Mathematical and computer modelling, vol. 48, pp. 56-68, 2008.

22. C. A. Borghi, A. Cristofolini, and M. Fabbri, "Study of the design 
model of a liquid metal induction pump," IEEE Trans. Magn., vol. 34, no. 4, pp. 2956-2959, 1998.

23. M. C. Miguel and J. M. Rubi, "On the dynamics of magnetic colloidal particles and holes," Physica A, vol. 231, pp. 288-294, 1996.

24. C. Holm and J. J. Weiss, "The structure of ferrofluids : A status report," Current Opinion in colloid and interface science, vol. 10, no. 4, pp. 133-140, 2005.

25. H. S. Lee and I. Nakatani, "On the chemical stability of ironnitride magnetic fluids in atmospheric conditions," Journal of Magnetism and Magnetic Materials, vol. 201, pp. 23-26, 1999.

26. G. S. Park and K. Seo, "New design of the magnetic fluid linear pump to reduce the discontinuities of the pumping forces," IEEE Trans. Magn., vol. 40, pp. 916-919, 2004.

27. N. Tiperi, "Overall characteristics of bearings lubricated ferrofluids," ASME J. Lubr. Technol., vol. 105, pp. 466-475, 1983.

28. J. Walker and J. Backmaster, "Ferrohydrodynamics thrust bearings," Int. J. Eng. Sci., vol. 17, pp. 1171-1182, 1979.

29. W. Ochonski, "The attraction of ferrofluid bearings," Mach. Des., vol. 77, no. 21, pp. 96-102, 2005.

30. H. Chang, C. Chi, and P. Zhao, "A theoretical and experimental study of ferrofluid lubricated four-pocket journal bearing," Journal of Magnetism and Magnetic Materials, vol. 65, pp. 372374, 1987.

31. Y. Zhang, "Static characteristics of magnetized journal bearing lubricated with ferrofluids," ASME J. Tribol., vol. 113, pp. 533538, 1991.

32. Q. Zhang, S. Chen, S. Winoto, and E. Ong, "Design of highspeed magnetic fluid bearing spindle motor," IEEE Trans. Magn., vol. 37 , no. 4 , pp. $2647-2650,2001$.

33. S. Chen, Q. Zhang, H. Chong, T. Komatsu, and C. Kang, "Some design and prototyping issues on a $20 \mathrm{krpm}$ hdd spindle motor with a ferro-fluid bearing system," IEEE Trans. Magn., vol. 37, no. 2, pp. 805-809, 2001.

34. T. Osman, G. Nada, and Z. Safar, "Static and dynamic characteristics of magnetized journal bearings lubricated with ferrofluid," Tribology International, vol. 34, pp. 369-380, 2001.

35. Z. Meng and Z. Jibin, "An analysis on the magnetic fluid seal capacity," Journal of Magnetism and Magnetic Materials, vol. 303, pp. e428-e431, 2006.

36. R. C. Shah and M. Bhat, "Ferrofluid squeeze film in a long 
bearing," Tribology International, vol. 37, pp. 441-446, 2004.

37. G. S. Park and S. H. Park, "Determination of the curvature of the magnetic fluid under external forces," IEEE Transactions on Magnetics, vol. 38, pp. 957-960, march 2002.

38. H. Chetouani, C. Jeandey, V. Haguet, H. Rostaing, C. Dieppedale, and G. Reyne, "Diamagnetic levitation with permanent magnets for contactless guiding and trapping microdroplets and particles in air and liquids," IEEE Trans. Magn., vol. 42, no. 10, pp. 3557$3559,2006$.

39. R. Ravaud, G. Lemarquand, V. Lemarquand, and C. Depollier, "Ironless loudspeakers with ferrofluid seals," Archives of Acoustics, vol. 33, no. 4, pp. 3-10, 2008.

40. F. Cunha and H. Couto, "A new boundary integral formulation to describe three-dimensional motions of interfaces between magnetic fluids," Applied mathematics and computation, vol. 199, pp. 7083, 2008.

41. G. Matthies and U. Tobiska, "Numerical simulation of normalfield instability in the static and dynamic case," Journal of Magnetism and Magnetic Materials, vol. 289, pp. 436-439, 2005.

42. A. Ivanov, S. Kantorovich, V. Mendelev, and E. Pyanzina, "Ferrofluid aggregation in chains under the influence of a magnetic field," Journal of Magnetism and Magnetic Materials, vol. 300, pp. e206-e209, 2006.

43. I. Tarapov, "Movement of a magnetizable fluid in lubricating layer of a cylindrical bearing," Magnetohydrodynamics, vol. 8, no. 4, pp. 444-448, 1972.

44. S. Miyake and S. Takahashi, "Sliding bearing lubricated with ferromagnetic fluid," ASLE Trans., vol. 28, pp. 461-466, 1985.

45. M. Miwa, H. Harita, T. Nishigami, R. Kaneko, and H. Unozawa, "Frequency characteristics of stiffness and damping effect of a ferrofluid bearing," Tribology Letter, vol. 15, no. 2, pp. 97-105, 2003. 